\title{
Magnetic Resonance for Wireless Power Transfer
}

\author{
Professor S.Y. Ron Hui \\ Department of Electrical Engineering \\ University of Hong Kong \& Imperial College London
}

\begin{abstract}
:
Magnetic resonance has been a cornerstone of non-radiative wireless power transfer (WPT) since the late $19^{\text {th }}$ century. Yet, there has been a misconception among some researchers who think magnetic resonance for WPT was developed recently. This article traces some early work of Tesla and other researchers related to the use of magnetic resonance in WPT. Some examples of magnetic resonance based WPT projects conducted by researchers in the biomedical and power electronics communities over last few decades are included. The article re-iterates two principles used in WPT and addresses their advantages and disadvantages. Some issues that may affect future trends of short-range and mid-range applications are addressed.
\end{abstract}

INTRODUCTION:

The scientific discoveries of Ampere's law and Faraday's law in the first half of the $19^{\text {th }}$ century provided the tools for many researchers to explore the properties of electricity and electromagnetic fields and waves. While Ampere and Faraday were experimentalists, J.C. Maxwell provided the mathematical equations for electromagnetic fields and waves. At the turn of the $20^{\text {th }}$ century, wireless power transfer research was actively pursued by early pioneers such as Tesla [1][2], Hutin and Leblanc [3]. Among these pioneers, Tesla undoubtedly made the most profound impact on short- and mid-range wireless power transfer (WPT) that affects even modern WPT applications. Tesla has been recognized as a highly visionary inventor who was well ahead of his time. After his death in January 7 1943, a memorial article [4] was published in the May issue of the Proceedings of the Institution of Radio Engineers (I.R.E). Tesla was described as " a catalyst in the realm of technology, a daring originator, and a dreamer on the grand scale". Besides his invention of the induction machines and wireless transmission of power and signals, this article states that:

“... he accurately described complex phenomena which in some instances were not fully understood for many years. And in one of them he envisioned that day when radio communication would truly make all the world one neighbourhood. He foresaw the time when a man might selectively summon his friend by a personal radio transmitter-receiver. And from the depths of a mine, the top of a mountain, or the vast reaches of an ocean, he would hear the voice of him whom he called." [4].

This extraordinary remark indicates that Tesla did foresee the mobile phone era (which became a reality in the 1990's). Tesla's technological innovations have transformed societies in the $21^{\text {st }}$ century and have been summarized in [5]. His inventions, to name a few, include induction machines, power transmission systems, radio systems, fluorescent discharge lamps, X-ray machines, the world's first remote-controlled boat, the world's first hydropower system and of course WPT. Nowadays, as people have been accustomed to the widespread use of electricity to a 
point that electricity is almost taken for granted, it would not be difficult to think of what modern lives would become without electricity for a prolonged period.

Some of Tesla's early work on high frequency systems with resonance phenomena was described in his lecture delivered to the Franklin Institute, Philadelphia in February1893 [6]. He had experimented with "condenser in series with the self-induction". This inductive-capacitive resonant circuit was fed by a high frequency alternator. To achieve a resonance condition, he mentioned that "both capacity (capacitance) and self-induction (inductance) were adjusted to suit the dynamo frequency". Tuned circuits has been a main theme shared among several of his research areas such as the transmitters and receivers of both radio signals and power. When talking about his work on the transmission of power through space in receiving the Edison Medal, Tesla commented "When there is no receiver there is no energy consumption anywhere" [7]. The reason is that the receiver circuit is a well turned circuit receptive to the transmission frequency. The idea of tuned frequency has also been the hallmark of the subsequent radio and TV signal transmission.

For magnetically coupled circuits, resonance refers to the energy oscillations between the magnetic field and the electric field. In electrical engineering terminology, it means energy oscillations between an inductive element (energy stored in the magnetic field) and a capacitive element (energy stored in the electric field). For loosely coupled windings, the leakage inductance is considerable. Capacitor can be added in series with the leakage inductance to reduce or cancel the effect of the leakage inductance. Consider two magnetically coupled winding systems as shown in Fig.1(a). For a loosely coupled system, the leakage inductance could be larger than the mutual inductance. Thus, the leakage reactance $\left(\omega L_{l k}\right)$ could limit the current flow to the output particularly when the operating frequency $(f=\omega / 2 \pi)$ is large. If a series capacitor is added and tuned at the resonance frequency $\left(f_{o}\right)$ of the $L C$ resonator for both the primary and secondary windings as shown in Fig. 1 (b), the leakage reactance can be cancelled and the impedance of the power flow path from the power source to the output of the seconding circuit can be minimized.

$$
j\left(\omega L_{l k}-\frac{1}{\omega C}\right)=0
$$

where $f_{o}=\frac{1}{2 \pi \sqrt{L_{l k} / C}}$ and $L_{l k}$ is the leakage inductance of the winding and $C$ is the resonance capacitor. Using the negative reactance of a capacitor to cancel the positive reactance of the leakage inductance is a type of "impedance matching" aiming at reducing the reactance of the power flow path in ac circuits. Impedance matching can be used for other purposes and is a topic that will be discussed in a later section. 


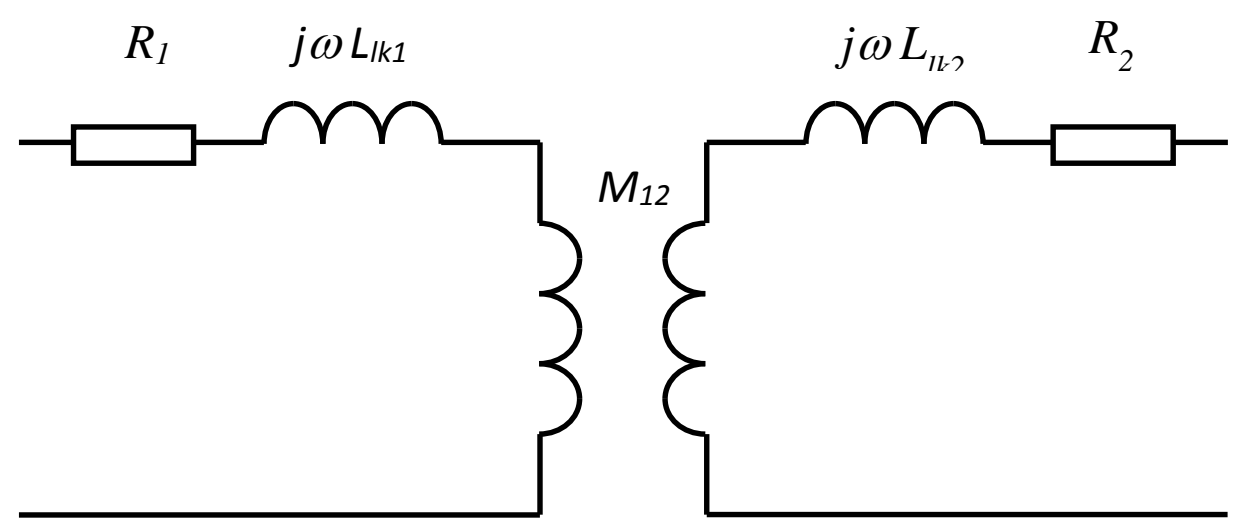

(a)

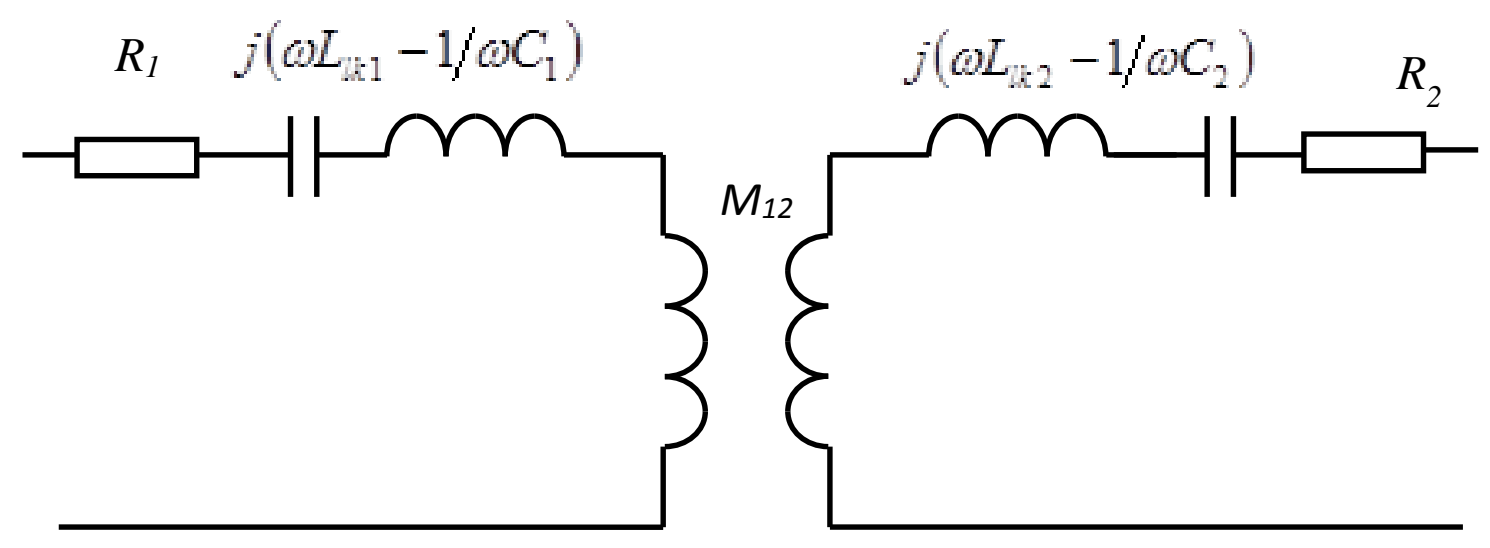

(b)

Fig.1 Equivalent circuit of (a) two magnetically coupled windings and (b) two magnetically coupled windings with series capacitor compensation

\section{SOME OF TESLA’S EARLY WORK ON MAGNETIC RESONANCE}

In 1893, Tesla described his high-frequency and resonance investigations in [6]. Inspired by the work of Heinrich Hertz and Oliver Lodge, he reported several experimental setups of his WPT study on using high-frequency oscillator for medical/therapeutic and other purposes [1]. Three of these setups are shown in Fig.2. Fig. 2(a) shows two magnetically coupled windings (Primary winding $P$ and Secondary winding $S$ ). The primary winding is connected in parallel with a condenser $C$ (capacitor) and is fed by a high-frequency generator $G$. The frequency was set in the range of 5-10 kHz. In the secondary winding, the two output terminals are labelled as $T$. In the description of this setup, Tesla added that "Two plates of large surfaces, forming an adjustable condenser, may be used for the purpose of synchronizing the secondary with the primary circuit".

Tesla further discussed the use of resonant circuits in Fig. 2(b), in which two parallel-connected condensers $C$ are connected in series with the primary winding $P$. The two ends of the secondary winding are connected to two sets of parallel plates tt and t't' of considerable surface (i.e. a form of 
variable capacitor). The voltage and current obtained from the two output terminals $\mathrm{T} T$ can be regulated by simply varying the distance between the two pairs of plates tt and t't' respectively. In modern terminology, this WPT system consists of a series-resonant primary winding and a seriesresonant secondary winding.

As Tesla continued to explain the operation of the system in Fig. 2(c) in [1], he emphasized the use of (i) high frequency, (ii) winding resistance as low as possible and (iii) the importance of establishing synchronism between the oscillations in the primary and secondary circuits. The $Q$ factor for an inductor is:

$$
Q=\frac{\omega L}{R}
$$

where $\omega$ is the angular frequency, $L$ is the inductance and $R$ is the winding resistance. These features are the factors essential to high quality factor (ie. High $Q$ factor) and magnetic resonance of the coupled windings. One of the short-range WPT applications demonstrated by Tesla was to power a lamp wirelessly as shown in Fig. 3.

(a)

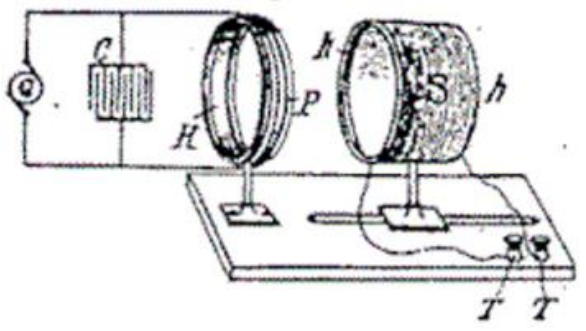

(b)

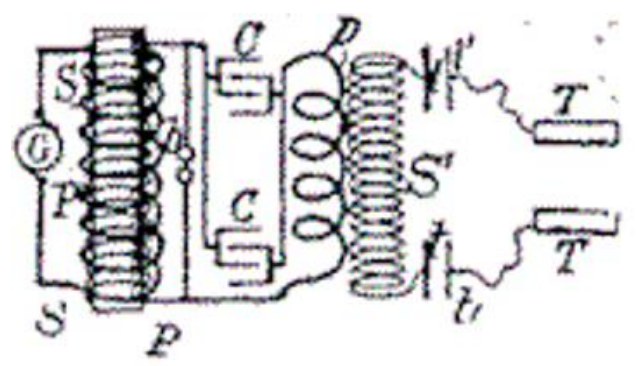

(c)

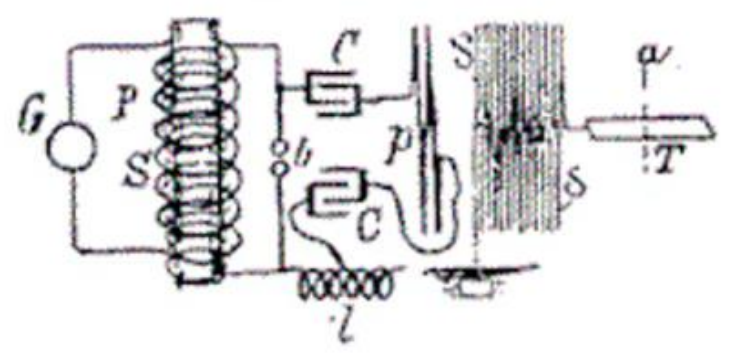

Fig.2 Three examples of WPT setups suggested by Tesla [1] 


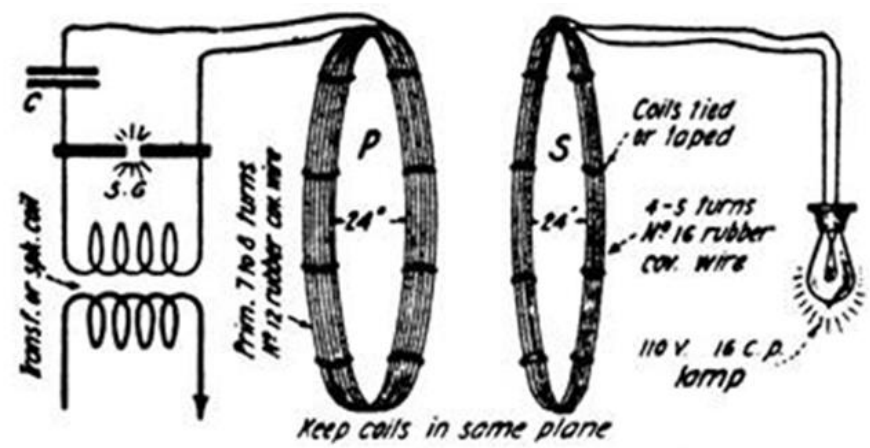

Fig. 3 An application of Tesla's WPT system for powering a lamp

The contributions of Tesla to the concept of using magnetic resonance were recognized in another memorial article published in August 1943 by L.P. Wheeler (The President of the Institute of Radio Engineers, 1943). In [8], Wheeler states that Tesla is entitled to either distinct priority or independent discoveries of:

a) The idea of inductive coupling between the driving and the working circuits

b) The importance of tuning both circuits, that is, the idea of an "oscillation transformer".

c) The idea of a capacitance loaded open secondary circuit.

The idea of oscillation transformer clearly refers to the use of magnetic resonance in magnetically coupled circuits. The use of magnetically coupled resonators operating under resonance conditions has been a hallmark of Tesla's WPT research.

\section{FACTORS RELATED TO MAGNETIC RESONANCE}

Many researchers have studied various aspects of technologies related to magnetic resonance of coupled circuits. Because of the large amount of works over the last century, this section only cites some relevant examples. Although it does not serve as a comprehensive review, it provides evidence that many WPT concepts considered today were studied in the past.

While Tesla was aware of the needs for using high frequency and winding with low resistance, Estill Green published an article about the story of $Q$ [9] in 1955. According to Green, it was K. S. Johnson who first used the symbol $Q$ in 1914 as the ratio of reactance to the effective resistance in a coil or a condenser. "Others before Johnson had made use of the ratio of reactance to resistance for either an inductor or a capacitor (to us modem parlance). But Johnson's role was to popularise this ratio and assign it to the contagious symbol $Q$. ." [9]. However, it is important to note in page 7 of [9] that "A rather spectacular recent achievement was the construction of an experimental ferrite inductor with a $Q$ of more than 1000." This indicates that the idea of using an inductor winding with $Q$ higher than 1000 was conceived over 60 years ago.

Magnetically coupled circuits was a hot research topic in early $20^{\text {th }}$ century as high frequency radio circuits were being investigated. The mathematical analysis of magnetically coupled oscillatory systems based on LC resonators was reviewed in 1916 by E.L. Chaffee [10]. He studied the general case of two coupled circuits with different resonance frequencies. The effects 
such as the amplitude and phase relationships of the primary and secondary current amplitudes and their phase shift of using the frequency (i) below, (ii) at and (iii) above the resonance frequency were examined.

\section{Energy Efficiency and kQ Product in Magnetically Coupled Resonant Circuits}

A biological heart uses typically $20 \mathrm{~W}$ to $35 \mathrm{~W}$ depending on whether it is in the resting or heavy exercise state. In 1961, John Schuder et al [11] studied power transfer for an artificial heart and used magnetic resonance technique to transfer power wirelessly through a closed chest wall of an animal. A series capacitor was used to compensate the leakage inductance in the secondary winding and the operating frequency was set at the resonance frequency of the $L C$ resonator. In their analysis, the significance of the product of the mutual coupling coefficient $k$ and the $Q$ factor of the winding was related to the power losses and thus energy efficiency of the system. It was found that power loss is inversely proportional to the $k Q$ product, indicating that the energy efficiency $(\eta)$ is proportional to the $k Q$ product.

$$
\eta \propto k Q
$$

For magnetically coupled windings with two different $Q$ factors,

$$
\eta \propto k \sqrt{Q_{1} Q_{2}}
$$

Equation (3) has two important meanings:

- The higher the $k Q$ product is, the higher the energy efficiency of the WPT system becomes.

- For loosely coupled systems in which the coupling coefficient $k$ is low, windings with high $Q$ factor should be used in order to increase the energy efficiency of the WPT systems. This feature applies to both short-range and mid-range applications.

These two points have been known for several decades in electrical engineering community. They are also in agreement with Tesla's suggestions of using high frequency and windings of low resistance.

\section{Non-Radiative WPT for Short-RAnge And Mid-RAnge ApPlications}

\section{A Short-Range Applications Based on Magnetic Resonance}

The substantial research on non-radiative WPT by Tesla and other scientists did not lead to widespread applications of WPT in the early half of the $20^{\text {th }}$ century. A major reason is the exponential reduction of the energy efficiency with the transmission distance of a WPT system. This issue was already pointed out in 1937 [12] and has also been reiterated recently in [13] and [14]. As stated by Tesla, (i) high frequency operation, (ii) low winding resistance and (iii) resonance operation are needed for efficient WPT. So it is necessary to have technologies that can operate at high-frequency with low ac resistance.

Two technologies namely (i) winding technology with low resistance at high frequency operation (such as Litz wires) and (ii) low-cost high frequency power conversion (such as power electronics) have influenced the progress of WPT.

- The concept of using multi-stranded wires to reduce high-frequency winding resistance was well documented in 1917 [15]. The commercial availability of Litz 
wires in the mid 1900's enabled the emergence of WPT activities for bio-medical applications in the 1960's.

- The maturity of power electronics in the 1980's has provided the needed technology to develop high-frequency power sources for modern WPT systems.

1) Examples of WPT research conducted by the bio-medical research communities

1961: John Schuder and his team reported the use of a pair of magnetically coupled circuits for WPT through the chest wall of an animal. The circuit involved is shown in Fig. 4. The series capacitor $C$ was used to compensate the leakage inductance. For a chest wall thickness of $3 \mathrm{~cm}$, the magnetically coupled circuit was operated at $400 \mathrm{kHz}$ corresponding to the resonance frequency of the secondary circuit. It was stated in [11] that Litz wire was used in this project to reduce the ac winding resistance. The windings were made in a spiral form with inner diameter of $1.5 \mathrm{~cm}$ and an outer diameter of $4 \mathrm{~cm}$. The coupling coefficient $k$ was 0.25 and the $k Q$ product is 50 (i.e. $Q$ factor $=200$ ). Short-term power of $50 \mathrm{~W}-69 \mathrm{~W}$ was successfully transmitted.

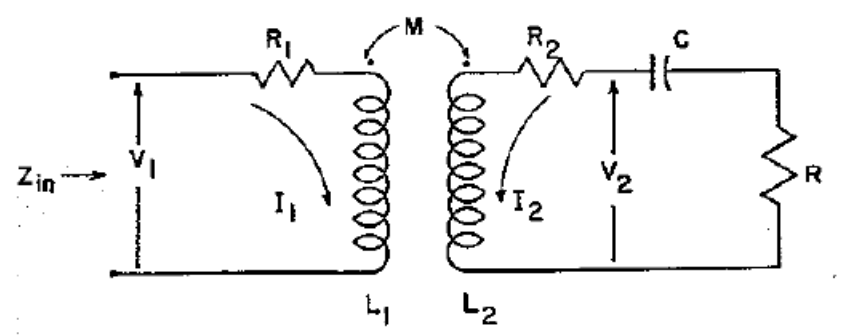

Fig. 4 Magnetically coupled circuit with a resonant tank in the receiver coil used in 1961 [11]

1968: Before the advent of power mosfets, the bipolar transistor based astable multivibrator (Fig. 5) was proposed by J. F. Fuller for WPT through a tissue barrier [16]. Fuller stressed the importance of the $Q$ factors and suggested the use of mica capacitors in the resonant tanks for $\mathrm{C} 1$ and $\mathrm{C} 2$ in Fig. 5. It is important to note that resonant circuits were used in both of the primary and secondary circuits (inside the dotted box in Fig.5). Magnetic resonance was used in this WPT. 


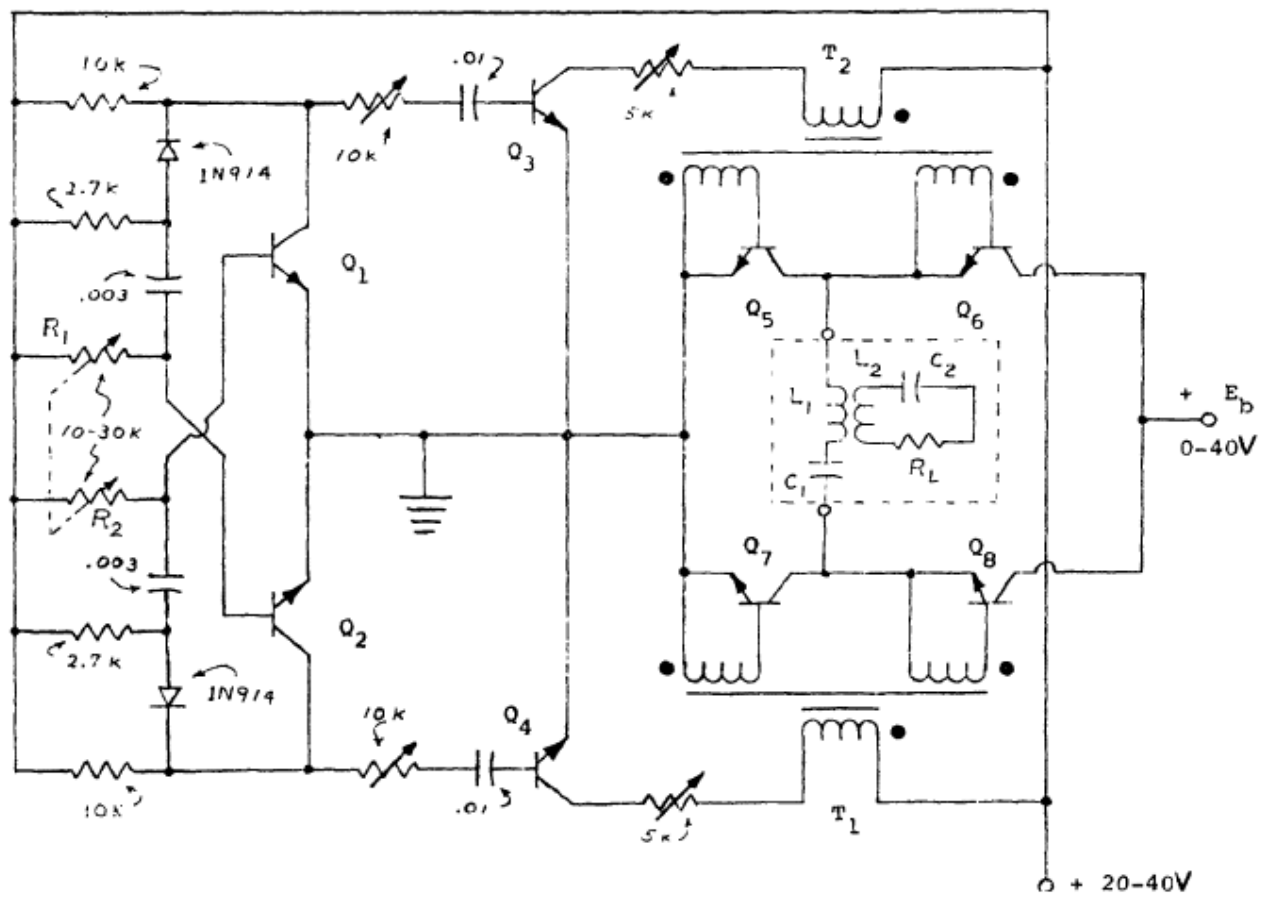

$Q_{1}-Q_{4}$ : Motorola 2N2193. $Q_{5}-Q_{8}$ : Motorola 2N3715.

Fig. 5 Transistor-based astable multivibrator used in 1968 for WPT [16]

1971: With a pair of much larger spiral coil made of Litz wires, John Schuder's team continued their research by transferring $1 \mathrm{~kW}$ through the skin of a dog (thickness of 7.5 $\mathrm{mm}$ ) [17]. It is interesting to note that they have considered the tissue conductivity, inductance and capacitance under an operating frequency of $480 \mathrm{kHz}$. The circuit structure is generally the same as that shown in Fig.4. The magnetically coupled circuits were operated in resonance mode.

2) Examples of WPT research conducted by the power electronics research communities

With the availability of power mosfets and resonant switching converters in the 1980s [18]-[20], research into the driving techniques and circuits for WPT system started to appear. The examples listed below adopt magnetic resonance based on $L C$ resonators.

1990: Bo Cho used a pair of magnetically coupled circuits operating at resonance mode for a transcutaneous energy transmission system as shown in Fig. 6 [21]. The coupling was fairly loose with a coupling coefficient of $k=0.1$.For an output power of $48 \mathrm{~W}$, an energy efficiency of $72 \%$ was achieved for an operating frequency of about $53 \mathrm{kHz}$. It should be noted that the "impedance matching" in the resonant tanks was implemented to reduce the effects of leakage inductance in the primary and secondary circuits. 


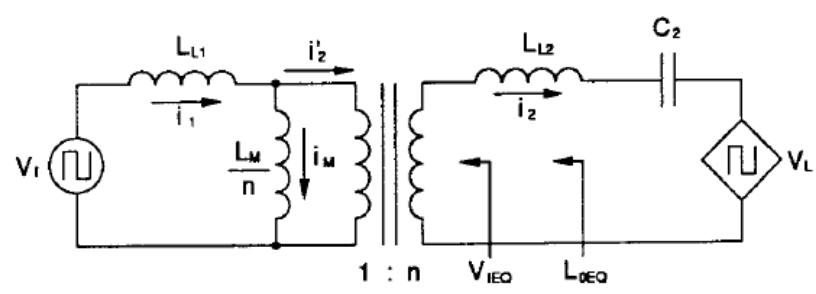

(a)

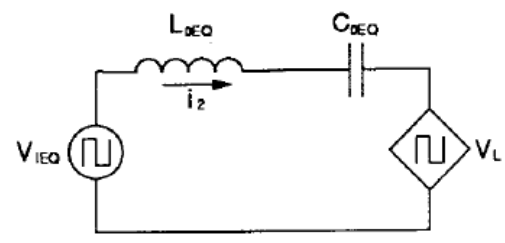

(b)

Fig. 6 (a) Magnetically coupled resonant circuit and (b) its equivalent circuit [21]

1994: A.W. Green and J. Boys presented their work in an inductively coupled power transmission (IPT) system based on resonant power converter and well-tuned (resonant) pick up coils (Fig. 7). Resonant tanks are used in the primary circuit (C1 and L1) and the secondary circuit (C2 and L2) [22].

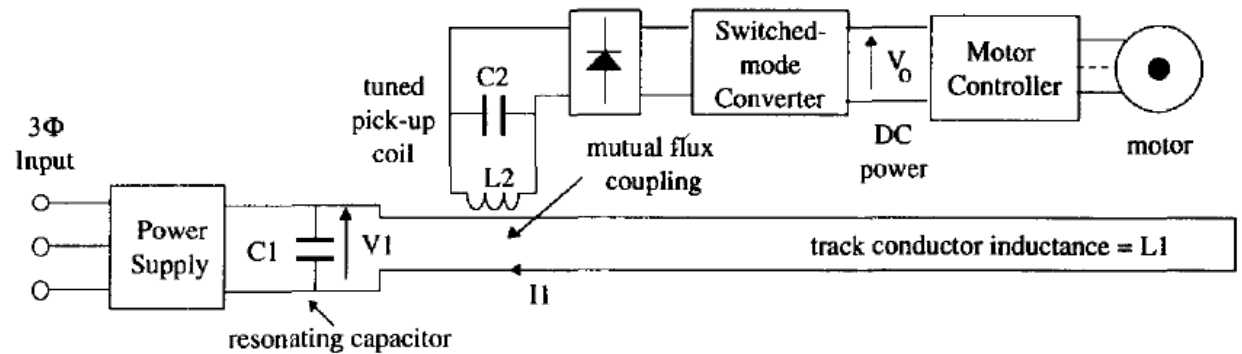

Fig. 7 Inductive power transmission system based on magnetic resonance [22]

1998: A small planar coreless transformer has been used for transferring power and signal. Energy was transferred from one planar spiral winding to another parallel planar winding without any magnetic core [23]. The diameter of the planar winding is $1 \mathrm{~cm}$ and each planar winding has 10 turns. The leakage inductances are the significant components of the equivalent circuit of the device. The stray capacitance is $3.5 \mathrm{pF}$ that can be used as part of the resonant circuit. But for the specific application in [23], an external capacitor $\mathrm{Cr}$ was used to reduce the resonance frequency. The energy stored in the magnetic field is set into resonance with an externally added capacitor $\mathrm{Cr}$ which stores energy in the electric field (Fig. 8). Resonance was used to maximize the performance of power and signal transfer across the gap between the magnetically coupled spiral coils. 


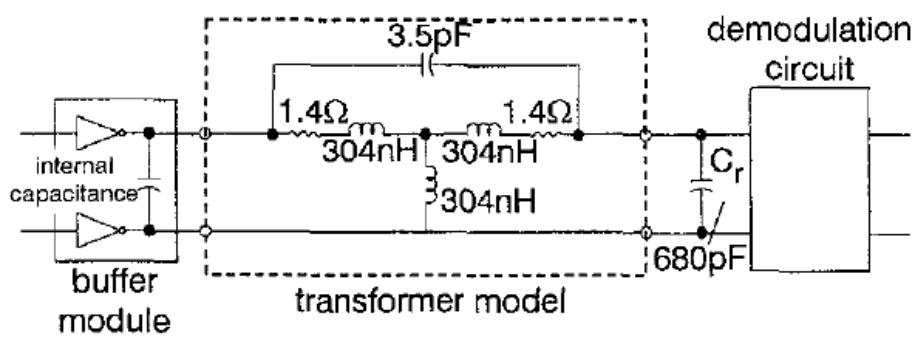

Fig. 8 Equivalent circuit of a planar coreless transformer based on magnetic resonance for power and signal transfer [23]

2000: With the dawn of mobile phone era in the 1900's, magnetic resonance has been used for wireless charging of mobile phones. Jang and Jovanovic used two magnetically coupled resonant circuits to form a WPT system, which is shown in Fig. 9 [24]. In fact, the use of magnetically coupled resonant circuits was not even considered as the novel aspect of this paper. It was used as a well-established power conversion technique for efficient energy transfer. The novelty of [24] is the soft-switching technique for further energy efficiency improvement on top of the magnetic resonance technique and the control design of this wireless charger for a wide input voltage range.

From the examples cited above, it is clear that magnetic resonance based on high $Q$ factors and well-tuned resonators has been a common practice among bio-medical and power electronics communities for many decades.

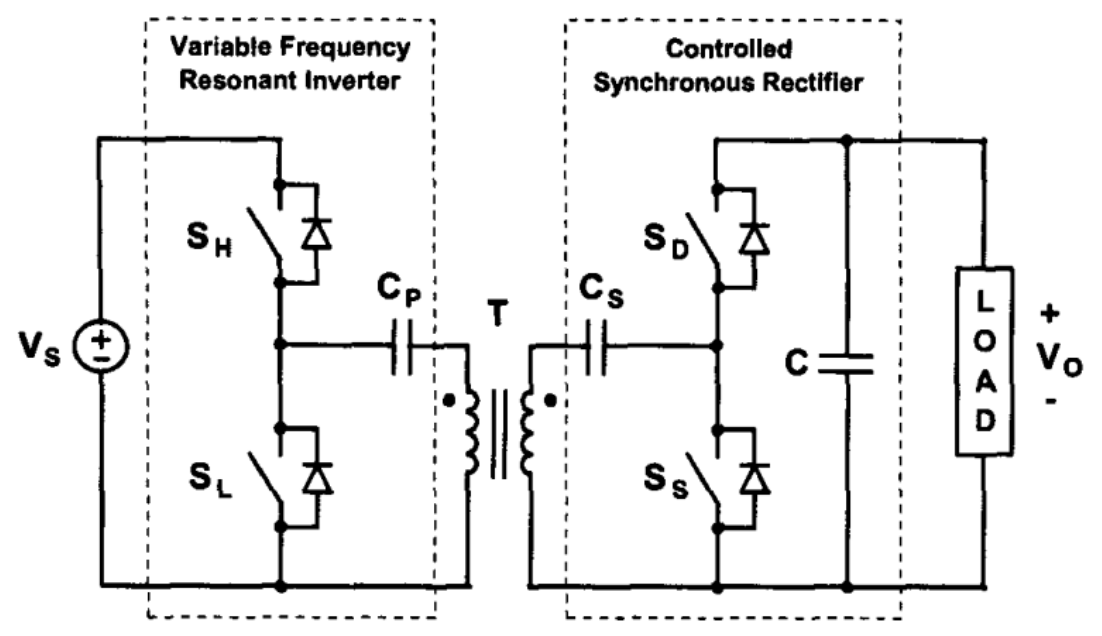

Fig. 9 Schematic diagram of inductive-coupled power stage with series-resonant inverter and controlled synchronous rectifier [24] 
Since Tesla described the use of WPT for powering a lighting device (Fig. 3), most of the 2coil WPT systems have been designed for short-range applications. In 1937, an interesting midrange 3-coil WPT system for powering a lamp was described [12]. Recently, several multiple-coil WPT systems have been reported. Before examples of mid-range WPT are described in this section, it is necessary to introduce the concepts of near-field coupling and mid-range WPT.

- Near-field distance is related to the wavelength of the electromagnetic wave and is usually defined as a distance within one wavelength $\lambda$ in a general sense. In a stricter sense, a distance of $\lambda / 2 \pi$ or $0.159 \lambda$ is considered as the range for near-field coupling.

- The concept of short-range and mid-range WPT is related to the ratio of the transmission distance $d$ and the coil dimension of the transmitter coil. In general, short-range coupling refers to a transmission distance less than or equal to the dimension of the transmitter structure. For a circular transmitter coil, $d \leq 2 r$ where $r$ is the radius of the coil. Mid-range usually refers to a distance of several times the dimension of the transmitter structure.

1900: One of the early WPT systems suggested by Tesla for mid-range application is shown in Fig. 10. It is a 4-coil system. The transmitter side has a driving loop $\mathrm{C}$ coupled to a transmitter coil A which significantly steps up the voltage for an elevated terminal D. Similarly, the receiver side has a load loop C' coupled to the receiver coil A' which is connected to an elevated terminal D'. Through electrical oscillation, current is discharged through air between D and D' by conduction.

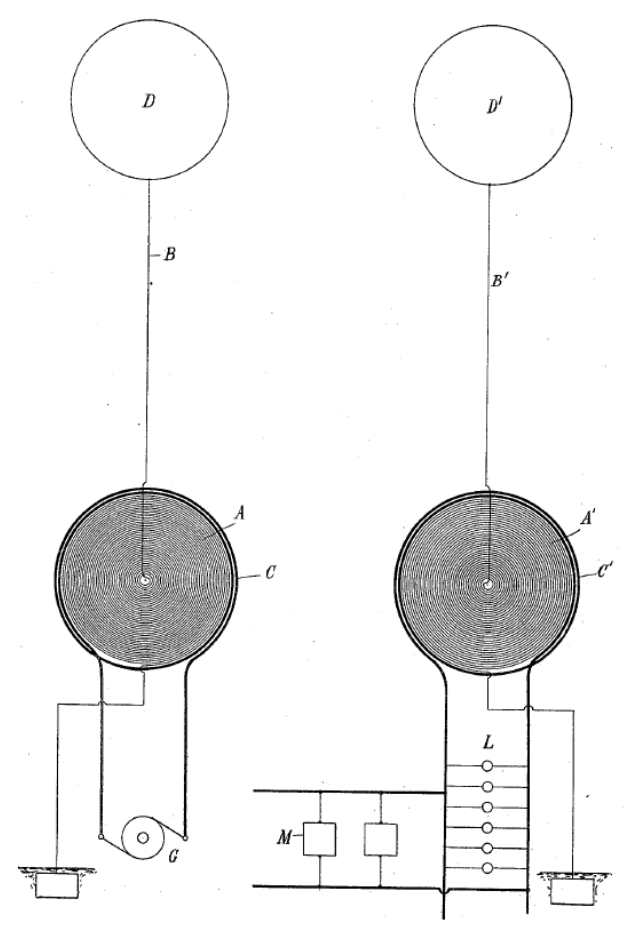

Fig. 10 A diagram of one of Tesla's wireless power experiments - by conduction [25]

1937: In August 1937, a cover story appeared in Short Wave \& Television Magazine [12] regarding an experiment deploying a Radio-frequency Power Transmitter to wirelessly power a 20W lamp over a distance of $10 \mathrm{ft}$ (i.e. $3 \mathrm{~m}$ ). The operating frequency was $100 \mathrm{MHz}$. An experimental setup (Fig. 11a) was installed in the Franklin Institute, Philadelphia (where Tesla had given the lecture). The unit on the left of Fig. 11a is the transmitter unit. This system is a 3-coil system and its circuit is shown in Fig. 11b. It includes a first coil magnetically coupled to a second coil that is used as an antenna. The transmitter circuit involves an oscillator driving a tuneable LC 
coil-resonator. The unit on the right of Fig. 11a is the receiver unit with a light bulb as the load. It consists of one receiving antenna which has tuneable inductance (using the left knob) and capacitance (using the right knob). According to [12], the variable condenser and inductor were used to demonstrate the idea of tuning the receiver resonator at the resonance frequency for receiving the power by the light bulb. For an operating frequency of $100 \mathrm{MHz}$, the wavelength $\lambda$ is about $3 \mathrm{~m}$, which is also the transmission distance. As the transmission distance is equal to one wavelength, the receiver was placed at the boundary of near-field and far field. The dimension of the antenna is $0.75 \mathrm{~m}$ and the transmission distance is $3 \mathrm{~m}$. Thus, this energy transmission could be considered as mid-range.

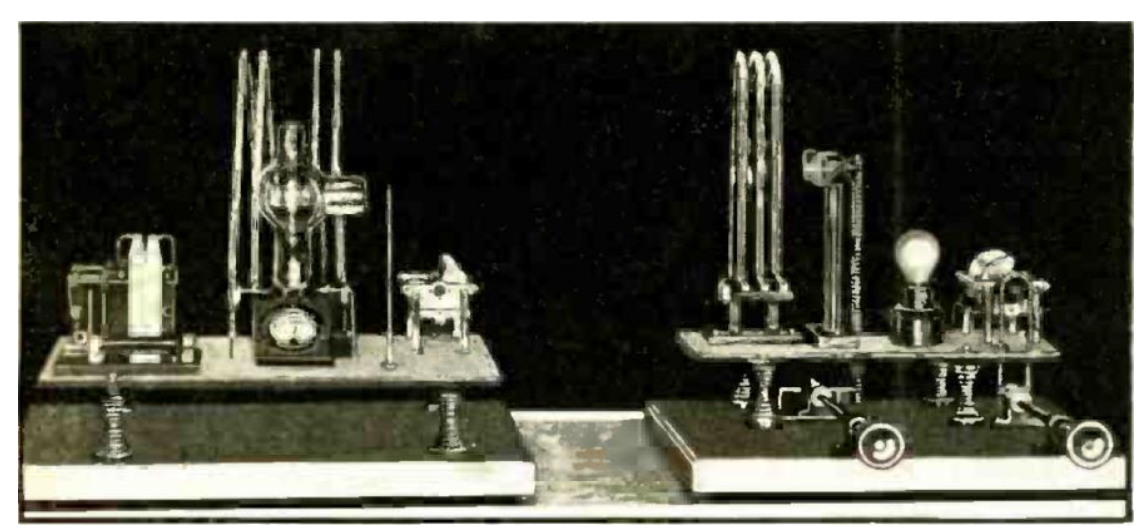

Fig. 11a Mid-range WPT setup in the Franklin Institute, Philadelphia - by radiation [12]

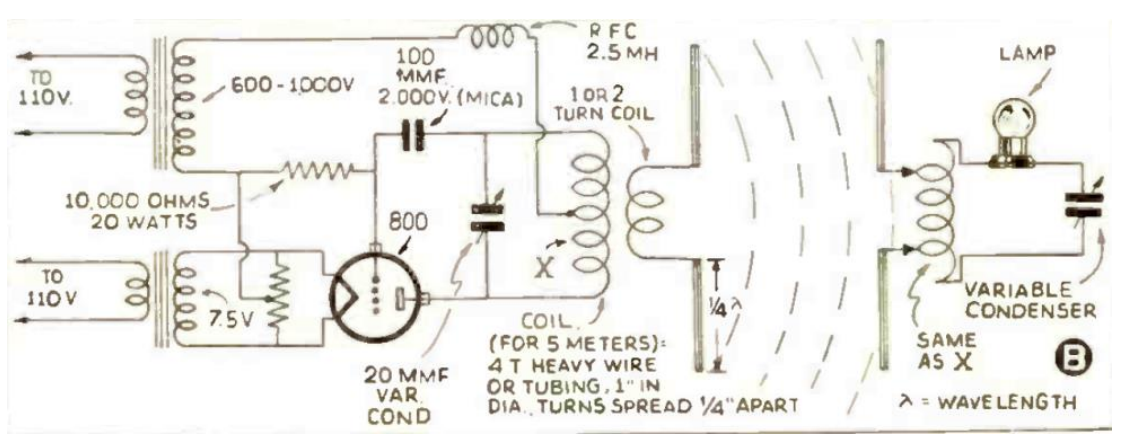

Fig. 11b Electric circuit for Fig. 11a [12]

2002,2006: The use of multiple magnetically coupled planar coil-resonators as a magnetoinductive waveguide (Fig. 12a) was proposed in [26][27]. The equivalent circuit system is shown in Fig. 12b. Each planar coil is made of a planar winding. In the analysis, each coil-resonator has a high $Q$-factor of $648(L=33 \mathrm{nH}, C=187 \mathrm{pF}$, and $R=20.5 \mathrm{~m} \Omega)$. The mean radius of the coil is $r=0.01 \mathrm{~m}$, the transmission distance between adjacent coil-resonators is $a=0.01 \mathrm{~m}$. The overall transmission distance is $(N-1) a$. Considering the mutual inductances among adjacent and nonadjacent coil-resonators, the system was operated at $63.87 \mathrm{MHz}$, the wavelength is $4.7 \mathrm{~m}$. Thus, this is a mid-range and near-field example. The research was followed up in 2006 [28] and the effects of $Q$-factor on the transmitted power was considered for $Q$ values of 100, 1,000 and 10,000 (for superconductor). As expected, a higher $Q$ leads to higher transmitted power. 
(a)

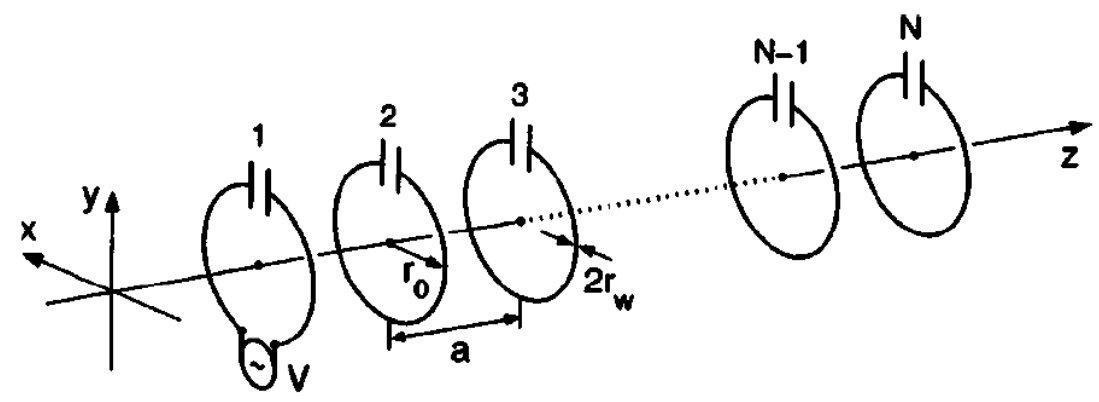

(b)

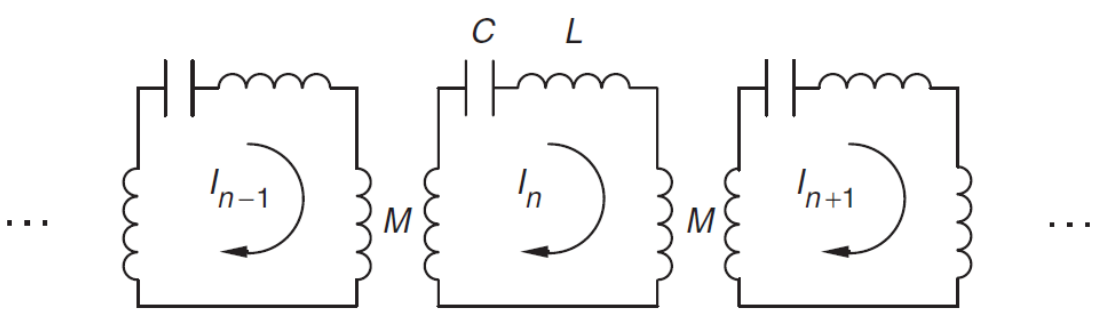

Fig. 12 (a) Magneto-inductive waveguide based on coil-resonators (- by magnetic resonance) and (b) its equivalent circuit [26]

2007: In 2007, a group of physicists from M.I.T. announced their project of wirelessly powering a 60W light bulb over a distance of $2 \mathrm{~m}$ [29][30]. The group emphasized the use of magnetic resonance instead of magnetic induction. The media publicity following their announcement has undoubtedly sparked off a lot of interest in WPT. Their work is based on a 4-coil system in which there are two resonators and two coupled coils as shown in Fig. 13(a). The use of a coupled coil to the resonator is similar to the transmitter stage of the 1937 experiment in Fig. 11(b). The analysis was conducted with the coupled mode theory. They identified the "strong coupling" regime [29] as:

$$
\kappa / \sqrt{\Gamma_{1} \Gamma_{2}}>>1
$$

where the symbol $\kappa$ is related to the mutual coupling coefficient $k$ as $\kappa=\left(\frac{\omega}{2}\right) k ; \Gamma_{1}=\frac{\omega}{2 Q_{1}}$ and $\Gamma_{2}=\frac{\omega}{2 Q_{2}}$ are the intrinsic loss rates of the first resonator and the second resonator respectively.

According to [29], this condition of (4) is "a regime of operation that has not been studied extensively". However, the term $\kappa / \sqrt{\Gamma_{1} \Gamma_{2}}$ of the coupled mode theory is equal to $k \sqrt{Q_{1} Q_{2}}$ in electric circuit theory, where $k$ is the mutual coefficient between the two coupled coils; $Q_{1}$ and $Q_{2}$ are the $Q$-factors of the two coil-resonators. Thus, $\kappa / \sqrt{\Gamma_{1} \Gamma_{2}}>>1$ simply means $k \sqrt{Q_{1} Q_{2}}>>1$ which has been a well-known concept in electrical engineering for several decades [16].

For mid-range application, the transmission distance is relatively large and so the mutual coupling coefficient $k$ is relatively small. For a small $k$, using a high $Q$ factor for each resonator would increase the energy efficiency as described in (3b). This point has been well known in electrical engineering for a century [1]. Regarding the suggestion of using resonance for efficient energy transfer [29][30], David Schneider commented in the IEEE Spectrum [31] that "It's not a new idea: Tesla's eponymous coils use that very same principle". 


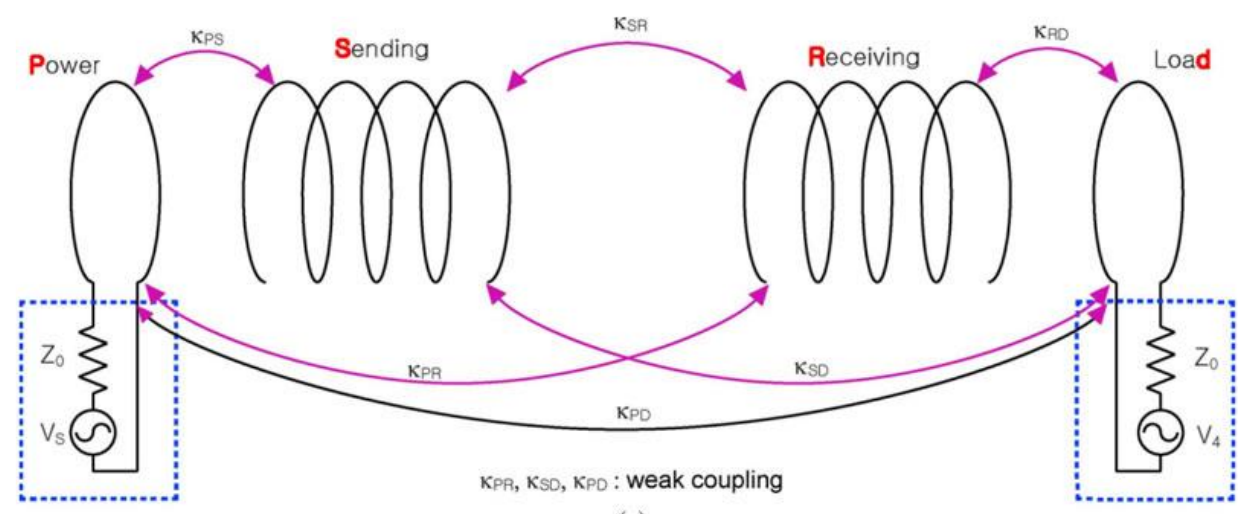

(a)
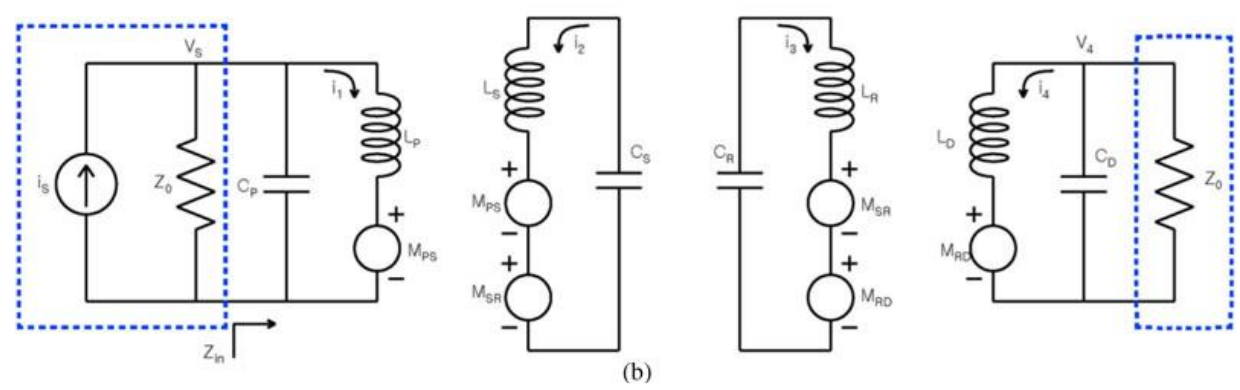

Fig. 13 (a) Schematic of the 4-coil WPT system proposed in [29] - by magnetic resonance and (b) its equivalent circuit

The 4-coil system in [29][30] has been analysed by several research groups using standard electric circuit theory [32]-[34]. Reference [32] provides a detailed analysis and an equivalent circuit of this system (Fig. 13). Basically, it shows that the operating principle of the 4-coil setup in [29][30] can be described by standard coupled circuit equations. The equivalent circuit is shown in Fig. 13(b). With an operating frequency at about $10 \mathrm{MHz}$, the stray capacitance of the resonator coil can be used as the resonance capacitor. The essence of this 4-coil WPT system lies in the provision of the two extra mutual inductance terms of the coupled loops (i.e. $k_{12}$ and $k_{34}$ ). The authors of [32] point out that the 4-coil system of [29][30] transmits power based on the impedance matching of the source impedance and the input impedance of the entire 4-coil WPT system. To achieve such impedance matching for maximum power transfer (i.e. $Z_{0}=Z_{\text {in }}$ in Fig. 13(b)), the following condition has to be met:

$$
\frac{k_{12} k_{34}}{k_{23}}=1
$$

The availability of $k_{12}$ and $k_{34}$ provides the flexibility in meeting this condition. For example, for a mid-range transmission distance between the two coupled resonators, their mutual coupling coefficient $k_{23}$ is small (e.g. 0.01 ), by adjusting $k_{12}$ and $k_{34}$ to be 0.1 , the condition of (5) can be met. The flexibility of tuning the coupling coefficients for satisfying (5) is a contribution of this 4-coil system, although such system involves no new scientific principle. But this is done at the expense of the system energy efficiency. Its system energy efficiency is $15 \%$, meaning that an input power of $400 \mathrm{~W}$ is needed to power a $60 \mathrm{~W}$ light bulb. Therefore, the 4-coil WPT system proposed in [29] and [30] is unsuitable for most applications in which energy efficiency is a concern. It may be useful for very low-power devices with charging power of less than one Watt. The low energy efficiency issue and mid-range WPT will be further addressed in the next sections. 
With many research papers on WPT published in recent years, one may ask a question of which WPT technique should be used. The fact that Radio-Frequency (RF) researchers and Power Electronics (PE) researchers use different approaches and terminologies is a source of confusion. This important question has been addressed in a critical review [35],[36], which classifies all WPT systems and techniques to be under either (i) the Maximum Energy Efficiency (MEE) Principle or (ii) Maximum Power Transfer (MPT) principle. Fig.14 (a) shows a basic circuit of a load powered by an ac power source. The source impedance is $R_{S}=j X_{S}$ and the load impedance is $R_{L}+j X_{L}$. The normalized power output and efficiency curve of this circuit are plotted in Fig. 14(b). It is very important to note that the operating point of MEE is different from that of MPT.

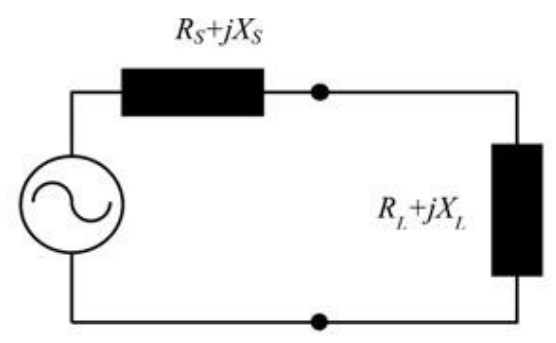

(a)

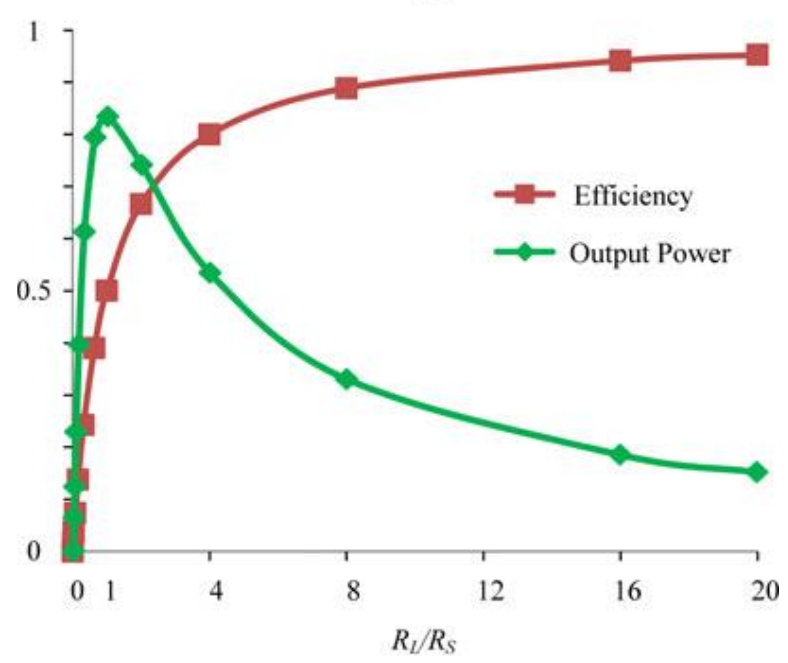

(b)

Fig. 14 (a) A basic ac electric circuit and (b) the corresponding energy efficiency and output power curves.

\section{A The Serious Problem of Ignoring Source Resistance in WPT}

Based on the "maximum power transfer theorem", maximum power can be transferred to the load when $R_{S}=R_{S}$ and $X_{S}=-X_{L}$. Under this MPT condition, half of the total power from the ac power source will be dissipated in the source resistance $R_{S}$. The ideal system energy efficiency is a ratio of the output power $P_{\text {out }}$ and input power $P_{i n}$ :

$$
\eta_{E}=\frac{P_{o u t}}{P_{\text {in }}}=\frac{i^{2} R_{L}}{i^{2}\left(R_{S}+R_{L}\right)}=\frac{R_{L}}{R_{S}+R_{L}}
$$


For meeting the MPT principle, $R_{S}=R_{S}$ and $\eta_{E}=0.5$. Therefore, for any WPT system that adopts "impedance matching" between the source impedance and the input impedance of the WPT system will never have system energy efficiency higher than 50\%. This is the theoretical limit that cannot be violated. This is the reason why the 4-coil WPT system proposed by the M.I.T team has a relatively low system efficiency of $15 \%$. It is noticed that many RF researchers are used to the MPT practice. They tend to use RF power amplifier with a source impedance of $50 \Omega$. It is a serious mistake to ignore the source resistance when the system energy efficiency is calculated.

On the contrary, PE researchers are used to the MEE concept of using minimum source resistance in the process of designing high-efficiency switched mode power supplies, magnetically coupled circuits and transformers. Under the MEE principle, the source resistance should be as small as possible. If $R_{S} \rightarrow 0$ in (6), $\eta_{E} \rightarrow 1$. Therefore, very high energy efficiency can be achieved. The "impedance matching" used by PE researchers usually refers to the design of the resonance capacitor to cancel the leakage inductance of the magnetically coupled coils. This is the reason why many switched mode power supplies, magnetically coupled circuits and transformer have system energy efficiency close to $100 \%$.

\section{B System Energy Efficiency or Transmission Energy Efficiency}

Another factor that may cause confusion is the efficiency definitions. Fig. 15 shows the schematic of a two-port network that can represent a WPT system. It is important to note that the system energy efficiency $\eta_{E}$ is different from the transmission efficiency $\eta_{T}$. They are defined as:

$$
\begin{gathered}
\eta_{E}=\frac{P_{3}}{P_{1}} \\
\eta_{T}=\frac{P_{3}}{P_{2}}
\end{gathered}
$$

where $P_{1}$ is total power provided by the ac power source (including the power loss in the source resistance), $P_{2}$ is the power available from the terminals of the ac power source (excluding the power loss in the source resistance) and $P_{3}$ is the power consumed by the load.

Some of RF researchers tend to use the S-parameter approach to analyse WPT systems by considering the energy in the form of transmitted and reflected waves. It is important to note that the S-parameter analysis does not include the power loss in the source resistance. Therefore, it is crucial for WPT researchers to understand the underlying principles of the analysis methods.

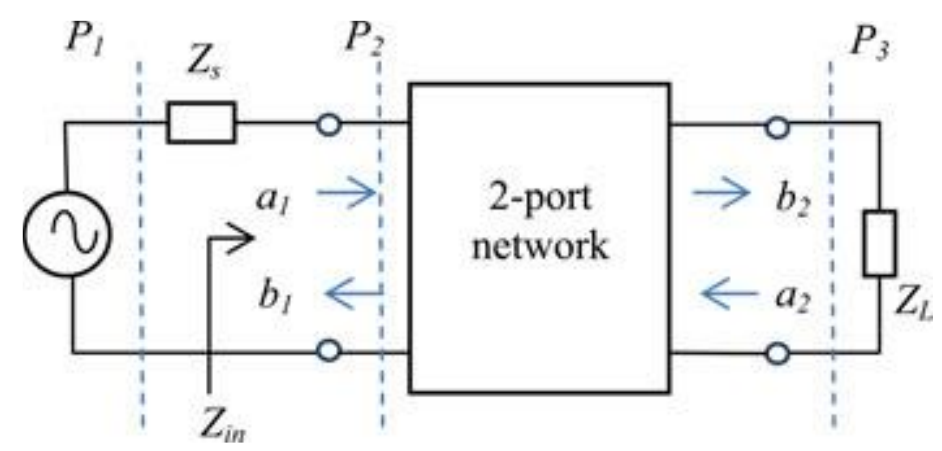

Fig. 15 Schematic of a 2-port network 
With the understanding of the MEE and MPT principles, one can easily determine which approach should be adopted for a specific application. The features of the two principles are summarized in Table I.

\begin{tabular}{|c|c|c|}
\hline & MEE & MPT \\
\hline Impedance matching & $\begin{array}{l}\text { To design the resonance } \\
\text { capacitor to compensate the } \\
\text { leakage inductance }\end{array}$ & $\begin{array}{l}\text { To match the input } \\
\text { impedance of the } \\
\text { WPT system with the } \\
\text { source impedance }\end{array}$ \\
\hline System efficiency & Can be higher than $50 \%$ & $\leq 50 \%$ \\
\hline Source resistance & As low as possible $\left(R_{S} \rightarrow 0\right)$ & $\begin{array}{l}\text { Dependent on the } \\
\text { power source }\end{array}$ \\
\hline Suitable power level & $\geq 1 \mathrm{~W}$ & $<1 \mathrm{~W}$ \\
\hline Applications & $\begin{array}{c}\text { Energy efficiency is a } \\
\text { priority }\end{array}$ & $\begin{array}{c}\text { Energy efficiency is } \\
\text { not a priority }\end{array}$ \\
\hline $\begin{array}{c}\text { Feature of a 2-coil } \\
\text { system }\end{array}$ & $\begin{array}{l}\text { High efficiency for short- } \\
\text { range, but very low } \\
\text { efficiency for mid-range } \\
\text { applications }\end{array}$ & $\begin{array}{l}\text { Low efficiency for } \\
\text { both short- and mid- } \\
\text { range applications }\end{array}$ \\
\hline $\begin{array}{l}\text { Feature of a 4-coil } \\
\text { system based on two } \\
\text { resonators and two } \\
\text { coupled coils [28][29] }\end{array}$ & & $\begin{array}{c}\text { Maximized } \\
\text { transmission distance } \\
\text { at the expense of } \\
\text { energy efficiency. }\end{array}$ \\
\hline $\begin{array}{l}\text { Feature of multi-coil } \\
\text { system based on relay } \\
\text { resonators [35][36] }\end{array}$ & $\begin{array}{c}\text { A good compromise } \\
\text { between energy efficiency } \\
\text { and transmission distance. }\end{array}$ & \\
\hline
\end{tabular}

\section{New Health AND SAFETy IsSUES FOR Mid-RANGe WPT}

While short-range WPT systems such as the wireless charging pads for portable consumer electronics usually use electromagnetic shields to confine the magnetic flux in order to avoid flux leakage, mid-range WPT systems may have to deal with new safety and health issues. New health concerns have recently been raised by quantum biologists. In 2014, practical evidence was presented in [37] to show that even a weak magnetic field of $10 \mu \mathrm{T}$ at $7 \mathrm{MHz}$ could enhance cell proliferation. (Note: The static magnetic field of the earth is about $45 \mu \mathrm{T}$.) Apparently, magnetic field at $7 \mathrm{MHz}$ could influence the electronic spins of cells to form radicals. The authors of [37] criticize the existing safety regulations which only consider the thermal stress on cells based on the specific absorption rate (Watt per kilogram of tissue) as inadequate. In their conclusion, they comment that "In contrast to the spin-pair mechanism, specific absorbed radiation (SAR) measurements of macroscopic tissue heating represent a nä̈ve approach to bio-magnetic RFinteractions because it ignores nanoscale physics and spin chemistry, which can potentially have profound biological effects." As a response to [37], the authors of [38] provide possible explanations for observed changes in growth rates of cancer cells and radical concentration upon exposures to magnetic fields below the ICES and ICNIRP reference levels. Perhaps it is time for 
international regulatory bodies to re-examine the existing health and safety regulations in view of the new evidence because $7 \mathrm{MHz}$ is close to the ISM band of $6.78 \mathrm{MHz}$ (which is an operating frequency being considered by some companies for WPT product developments).

For mid-range applications, one challenge is to shield any foreign lossy object in the path of the WPT. In 2012, there was a suggestion of using a conducting surface and a layer of magnetic material to shape the magnetic field [39] in order to avoid lossy objects. It should be noted that the use of a double-layer electromagnetic shield comprising a layer of conducting material and a layer of soft magnetic materials for confining the magnetic field was previously described in 2002 [40] and [41]. If weak magnetic field of frequency close to $7 \mathrm{MHz}$ could induce health hazards, not only lossy objects but also human bodies should be shielded from such magnetic field.

\section{CONCLUSIONS}

This article traces some historical works of WPT and shows that many important concepts such as high $Q$ factor, magnetic resonance, magnetically coupled resonators are not recent developments. Many research pioneers over the past century have laid down these scientific principles for WPT. In particular, magnetic resonance for efficient wireless energy transfer has been widely used in many WPT projects for many decades. The classification of the maximum energy efficiency principle and maximum power transfer principle for WPT systems should make it easy for researchers and engineers to distinguish the nature of different WPT systems and help them decide which principle should be adopted for a specific application. The importance of including the power loss in the source resistance of the power source is emphasized because it is a crucial factor that determines the overall energy efficiency of the entire system. It is important to note that essentially all of the transmitter circuits and receiver circuits specified in the $Q i$ standard [42] are magnetically coupled resonant circuits. The use of magnetic resonance including the maximization

of the $k \sqrt{Q_{1} Q_{2}}$ product for efficient energy transfer has been common practice in electrical engineering for many decades. Recent concerns about biological effects of low-level $7 \mathrm{M} \mathrm{Hz}$ magnetic field on the cell proliferation raised by the quantum biologists should be taken seriously as such biological effects may have safety and health implications on future mid-range WPT applications. Short-range WPT systems usually have the benefit of having the magnetic flux enclosed by EM shields, and so do not have such EM field exposure issues.

\section{References}

[1] Nikola Tesla, "High frequency oscillators for electro-therapeutic and other purposes", The Electrical Engineer. Vol. XXVI. November 17, 1898. No. 550.

[2] Nikola Tesla, "Transmission of electrical energy without wire", Electrical World and Engineer, March 5, 1904 [online] www.tfcbooks.com/tesla/

[3] M. Hutin and M. Leblanc, "Transformer system for electric railways", US patent 527857 , Oct. 23, 1894

[4] "Nikola Tesla 1857 - 1943", May 1943, Proceedings of the I.R.E., pp: 194

[5] R. Lomas, "The Man Who Invented the Twentieth Century - Nikola Tesla - Forgotten Genius of Electricity”, U.K., Headline Book Publishing, 1999

[6] Nikola Tesla, "On light and other high frequency phenomena", Lecture Delivered before the Franklin Institute, Philadelphia, February 1893, and before the National Electric Light Association, St. Louis, March 1893. [online] www.tfcbooks.com/tesla/ 
[7] Minutes of the annual meeting of the American Institute of Electrical Engineers, held at the Engineering Society Building, New York City, Friday Evening, May 18, 1917

[8] L.P. Wheeler, "Tesla's contribution to high frequency", Electrical Engineering, New York, August 1943, pp: 355-357

[9] Estill I. Green, "The story of Q", Bell Telephone System - Technical Publication Monograph 2491, pp: 1-11 (also published in American Scientist, Vol. 43, Oct. 1955, pp: 584-594)

[10] E.L. Chaffee, "Amplitude Relations in Coupled Circuits", Proceedings of the Institute of Radio Engineers, Year: 1916, Volume: 4, Issue: 3, Pages: 283 - 300

[11] J.C. Schuder, H.E. Stephenson and J.F. Townsend, "High level electromagnetic energy transfer through a closed chestwall, IRE Int. Conv. Rec., vol. 9, 1961, pp: 119-126

[12] Hugo Gernsback (Editor) "Lighting lamp by S-W- Radio", Short Wave \& Television, August 1937, pp: 166 and 191

[13] E. Waffenschmidt and T. Staring, "Limitation of inductive power transfer for consumer applications", European Power Electronics conference, 2009, pp: 1-10.

[14] J. O. Mur-Miranda, G. Fanti, Y. Feng, K. Omanakuttan, R. Ongie, A. Setjoadi and N. Sharpe, "Wireless power transfer using weakly coupled magnetostatic resonator", IEEE Energy Conversion Congress and Exposition (ECCE), 2010, pp: 4179-4186

[15] G.W. Howe, "The high-frequency resistance of multiply-stranded insulated wire", Proceedings of the Royal Society of London, Series A, Vol. 93, No. 654, Sept. 1, 1917, pp: 468-492

[16] J.W. Fuller, “Apparatus for efficient power transfer through a tissue barrier", IEEE Transaction on Biomedical Engineering, Vol.BME-15, Jan. 1968, pp: 63-65

[17] J.C. Schuder, J.H. Gold and H.E. Stephenson, "An inductively coupled RF system for the transmission of $1 \mathrm{~kW}$ of power through the skin", IEEE Transactions on Biomedical Engineering, Vol. 18, No.4, July 1971, pp: 265-273

[18] V. Veroerian and S. Cuk, "Small-signal analysis of resonant converters", IEEE Power Electroincs Specialists Conference Record, 1983, pp: 269-282

[19] V. Vorperian, "Analysis of resonant converters", Ph.D thesis, California Institute of Technology, Pasadena, USA, May 1984.

[20] R. Oruganti and F.C. Lee, "Resonant power processors, Part-I - State-space analysis", IEEE Transactions on Industry Applications, vol. IA-21, No. 6, 1985, pp: 1453-1460

[21] A. Ghahary and B. Cho, "Design of a transcutaneous energy transmission system using a series resonant converter", 1990

[22] A.W. Green and J.T. Boys, "10k Hz inductively coupled power transfer - concept and control”, 1994 Power Electronics and Variable Speed Drives Conference, 1994, pp: 694-699

[23] S.Y.R. Hui, S.C. Tang and H. Chung, "Coreless printed-circuit-board transformer for signal and energy transfer, Electronics Letters, Vol. 34, No. 11, 28 May, 1998, pp: 1052-1054

[24] Yungtaek Jang; M.M. Jovanovic, “A contactless electrical energy transmission system for portable-telephone battery chargers", Twenty-second International Telecommunications Energy Conference, 2000. INTELEC., Pages: 726 - 73

[25] N. Tesla, "System of Transmission of Electrical Energy", US patent 645576, March 20, 1900.

[26] E. Shamonina, V.A. Kalinin, K.H. Ringhofer and L. Solymar, "Magneto-inductive waveguide", Electronics Letters, Vol. 38, No.8, 11 April 2002, pp: 371-373

[27] E. Shamonina, V.A. Kalinin, K.H. Ringhofer and L. Solymar, "Magneto-inductive wavegs in one, two and three dimensions", Journal of Applied Physics, Vol. 92, No. 10, 15 Nov.2002, pp: 6252-6261 
[28] R. Syms, E. Shamonina and L. Solymar, "Magneto-inductive waveguide devices", IEE Proceedings of Microwave, Antennas, Propagation, Vol. 153, No.2, April 2006, pp: 111-121

[29] A. Kurs, A. Karalis, R. Moffatt, J. D. Joannopoulos, P. Fisher and Marin Soljac `ic', "Wireless Power Transfer via Strongly Coupled Magnetic Resonances", Science, Vol. 317, July 2007, pp: 83-86

[30] A. Karalis, J. D. Joannopoulos, and Marin Soljac `ic', "Efficient wireless non-radiative midrange energy transfer", Annals of Physics, 27 April 2007, pp: 34-48

[31] D. Schneider, "A critical look at wireless power transfer", IEEE Spectrum, Posted 30 Apr 2010

[32] S. Cheon, Y.H. Kim, S.Y. Kang, M. L. Lee, J.M. Lee, and T. Zyung, "Circuit-model-based analysis of a wireless energy-transfer system via coupled magnetic resonances", IEEE Trans. Industrial Electronics, vol. 58, no. 7, pp. 2906-2914, Jul. 2011

[33] C.J. Chen, T.H. Chu, C.L. Lin, and Z.C. Jou, "A study of loosely coupled coils for wireless power transfer", IEEE Trans. Circuitsand Systems - II: Express Briefs, vol. 57, no. 7, pp. 536-540, Jul. 2010.

[34] M. Kiani, and M.. Ghovanloo, "The circuit theory behind coupled-mode magnetic resonance-based wireless power transmission," IEEE Trans. Circuits Systems - I, vol. 59, no. 8, pp. 1-10, Aug. 2012

[35] S.Y.R. Hui, W.X. Zhong and C.K. Lee, "A critical review of recent progress in mid-range wireless power transfer", IEEE Transactions on Power Electronics, Vol. 29, No. 9, Sept. 2014, pp: 4500-4511

[36] W.X. Zhong, C.K. Lee and S.Y.R. Hui, "Recent progress of mid-range wireless power transfer", IEEE Energy Conversion Congress and Exposition (ECCE), 25-20 Sept. 2012, Raleigh, N.C., USA, 2012, Page(s): 3819 - 3824

[37] R. J. Usselman, I. Hill, D. J. Singel, Carlos F. Martino, "Spin Biochemistry Modulates Reactive Oxygen Species (ROS) Production by Radio Frequency Magnetic Fields”, PLOS, Volume 9, Issue 3, March 2014, pp:1-12

[38] Frank Barnes and, Ben Greenebaum, "Magnetic Field Effects on Biology and Potential Health Effects Below the ICES and ICNIRP Reference Levels", IEEE Wireless Power Transfer conference, Boulder, N.C., USA, May, 2015, Paper T5.8, pp: 1-4

[39] A. Karalis, A. Kurs, K. Kulikowski, K. Hall, M. Soijacic and M. Kesler, "Wireless energy transfer using field shaping to reduce loss", US patent 8,304,935, Nov. 6, 2012

[40] S.Y.R. Hui and S.C. Tang, "Planar printed-circuit-board transformers with effective electromagnetic interference (EMI) shield, US patent 6,501,,364, Dec. 31, 2002.

[41] S.C. Tang, S.Y.R Hui and H. Chung, 'Evaluation of the Shielding Effects on Printed-CircuitBoard Transformers using Ferrite Plates and Copper Sheets', IEEE Transactions on Power Electronics, Vol.17, No.6, Nov. 2002, pp.1080-1088.

[42] Qi, System Description, Wireless Power Transfer, Volume I: Low Power, Part 1: Interface Definition, Version 1.1.2, June 2013 [Wireless Power Consortium website, August 2015] 